\title{
RISCO OCUPACIONAL ENTRE PROFISSIONAIS DE SAÚDE DO SERVIÇO DE ATENDIMENTO MÓVEL DE URGÊNCIA - SAMU
}

Hillda Dandara Carvalho Santos Leite ${ }^{1}$, Maycon Teyllon Rodrigues de Carvalho², Suelky Lilyan da Silva Cariman³, Eronice Ribeiro de Morais Araújo ${ }^{4}$, Naldiana Cerqueira Silva ${ }^{5}$, Amália de Oliveira Carvalho

Objetivo: avaliar os riscos ocupacionais referidos pelos profissionais de saúde do SAMU de Teresina - PI. Metodologia: estudo do tipo descritivo, transversal, realizado com 69 profissionais de saúde do SAMU de Teresina - PI mediante aplicação de um questionário com perguntas fechadas. Resultados: entre os riscos físicos, $87 \%$ (60) destacaram os ruidos da sirene; $79,7 \%$ (55) afirmaram a colisão de automóveis como principal risco de acidente; 79,7\% (55) informaram ser o levantamento de peso o principal risco ergonômico; 89,9\% (62) apontaram o sangue como o principal risco biológico; 63,8\% (44) afirmaram que a poeira é o principal risco químico, e 82,6\% (57) destacaram o estresse como principal risco psicossocial. Conclusão: a gestão participativa e o aperfeiçoamento profissional poderão minimizar a exposição dos servidores desse serviço a tais riscos.

Descritores: Riscos ocupacionais, SAMU, Enfermagem

\section{OCCUPATIONAL RISK AMONG HEALTH PROFESSIONAL OF THE MOBILE SERVICE OF EMERGENCY CARE - SAMU}

Objective: evaluate the occupational risks referred to by health professionals of the Mobile Service of Emergency Care, TeresinaPI. Methodology: descriptive and transversal study, conducted with 69 health professionals from SAMU Teresina - PI. Data collection was performed by a questionnaire with closed questions. Results: Among the physical risks, $87 \%$ (60) highlighted noise of the siren; 79,7 \% (55) said the car crash as a major risk of accidents; $79.7 \%$ (55) reported to be weight lifting the main ergonomic risk; $89.9 \%$ (62) showed the blood as the main biological risk; $63.8 \%$ (44) stated that the dust is the main chemical risk and $82.6 \%$ (57) highlighted stress as main psychosocial risk. Conclusion: Participatory management and professional development can minimize exposure of the servers that service to such risks

Descriptors: Occupational risks, Emergency Medical Services, Nursing

\section{RIESGOS LABORALES ENTRE PROFESIONALES DE UN SERVICIO MÓVIL DE URGENCIA- SAMU}

Objetivo: evaluar los riesgos profesionales mencionados por los profesionales de la salud SAMU de Teresina - PI. Metodologia: Estudio descriptivo, transversal, realizado con 69 profesionales de la salud de SAMU Teresina - PI, un cuestionario con preguntas cerradas. Resultados: entre riesgos física 87\% (60) puso de relieve que el ruido de la sirena; 79,7 \% (55) dijo que el accidente de coche como el principal riesgo de accidentes; 79,7 \% (55) informó de que el levantamiento de pesas el principal riesgo ergonómico; 89,9 \% (62) mostró la sangre como principal riesgo biológico; 63,8 \% (44) declaró que el polvo es el principal riesgo químico y el 82,6 \% (57) destacó el estrés psicosocial como el riesgo principal. Conclusión: La gestión participativa y el desarrollo profesional pueden minimizar la exposición de los servidores que dan servicio a este tipo de riesgos.

Descriptores: Riesgos laborales, Servicios Médicos de Urgencia, Enfermería

${ }^{1}$ Acadêmica de Enfermagem do 10ㅇs semestre da Faculdade Integral Diferencial (FACIDIDEVRY). E-mail: hilldadandara@hotmail.com

${ }^{2}$ Acadêmico de Enfermagem do 10 ㅇ semestre da Faculdade Integral Diferencial (FACIDIDEVRY).

${ }^{3}$ Acadêmica de Enfermagem do 10ㅇs semestre da Faculdade Integral Diferencial (FACIDIDEVRY).

${ }^{4}$ Mestre em Enfermagem. Professora do curso de Enfermagem da Faculdade Integral Diferencial (FACIDIDEVRY).

5 Mestre em Enfermagem. Professora do curso de Enfermagem da Faculdade Integral Diferencial (FACIDIDEVRY)

${ }^{6}$ Mestre em Enfermagem. Professora do curso de Enfermagem da Faculdade Integral Diferencial (FACIDIDEVRY). 


\section{INTRODUÇÃO}

A Organização Internacional do Trabalho estimou que 2,34 milhões de pessoas morrem anualmente no mundo em decorrência de acidentes e doenças relacionados com o trabalho, sendo 2,02 milhões (86,3\%) causados por Doenças Profissionais e 321 mil em consequência de Acidentes de Trabalho. São 6.300 mortes diárias relacionadas ao trabalho, 5.500 causadas por Doenças Profissionais, números esses inaceitáveis, os quais indicam que ações devem ser intensificadas em busca do trabalho adequadamente remunerado, exercido em condições de liberdade, equidade e segurança, além de ser capaz de garantir uma vida digna às pessoas ${ }^{(1)}$.

Os riscos ocupacionais advindos de mudanças tecnológicas e sociais resultantes da globalização, afetam significativamente a saúde dos trabalhadores, ainda que alguns dos riscos tradicionais tenham diminuido devido a maior segurança, melhor regulamentação e maiores recursos técnicos empregados. Simultaneamente, surgem novos tipos de Doenças Profissionais, oriundas de riscos emergentes ocasionados por condições ergonômicas deficientes, exposição à radiação eletromagnética e riscos psicossociais ${ }^{(1)}$

No caso específico dos trabalhadores de saúde do Serviço de Atendimento Móvel de Urgência (SAMU), em decorrência de suas peculiaridades, esses profissionais enfrentam situações que os deixam mais vulneráveis a riscos ocupacionais, tais como: acesso difícil às vítimas, insegurança na cena do acidente, realização de procedimentos com o veículo estático ou em movimento. Deve-se ressaltar, ainda, que tais profissionais atuam nos mais variados locais de atendimento e, em grande parte, sob condições desfavoráveis de luminosidade, chuva, calor, frio, fluxo de veículos, falta de higiene, presença de animais, pessoas agressivas, tumultos sociais, e tais fatores são condições que diferenciam esse trabalho daquele do ambiente hospitalar ${ }^{(2)}$

Os profissionais de saúde do SAMU se submetem mais a riscos ocupacionais do que aqueles servidores de saúde que trabalham no ambiente hospitalar, pois fornecem atendimento a vitimas em diversos locais e nas mais variadas circunstâncias. Sendo assim, torna-se evidente que os mesmos encontram-se constantemente vulneráveis a todo tipo de risco ocupacional, entre eles: físicos, químicos, acidente, biológicos, ergonômicos e psicossociais.

Os riscos ocupacionais são definidos como todo acidente que coloque o trabalhador em situação vulnerável e possa afetar sua integridade e seu bem estar físico e psíquico. Nessa perspectiva, o risco físico acontece quando o profissional fica exposto aos agentes explosivos, radiações, altas temperaturas e ruídos; o químico quando entra em contato com produtos tóxicos, seja manipulando medicamentos ou desinfetando a ambulância; de acidente, devido ao espaço limitado e acomodação física da ambulância, iluminação inadequada, assistência à vítima com a ambulância em movimento; biológico quando entra em contato com microrganismos patógenos ${ }^{(3)}$.

Risco ergonômico é quando se submetem a movimentos bruscos e repetitivos, peso excessivo, estresse, posições incômodas e prolongadas; e os riscos psicossociais englobam constante cobrança de produtividade e imposição de tarefas pela chefia, jornadas de trabalho extenuantes, padrão do sono irregular, monotonia, além de outras situações causadoras de estresse psíquico (3).

Considerando que os profissionais de saúde do SAMU estão vulneráveis aos riscos ocupacionais, em decorrência das condições inerentes ao próprio atendimento préhospitalar, este estudo traçou como objetivo avaliar os riscos ocupacionais referidos pelos profissionais de saúde do SAMU de Teresina - PI.

\section{METODOLOGIA}

Estudo descritivo, transversal e de abordagem quantitativa, realizado com os profissionais de saúde do SAMU do município de Teresina-PI. A população foi de 69 profissionais, sendo 16 enfermeiros, 33 técnicos de enfermagem e 20 médicos, que trabalhavam há mais de um ano no serviço. Foram excluídos aqueles que se recusaram a participar da pesquisa ou que estavam de férias, licença maternidade, prêmio ou médica.

A coleta de dados aconteceu entre janeiro e fevereiro de 2016. Utilizou-se um questionário estruturado, adaptado do estudo de Fernandes ${ }^{(1)}$, possibilitando traçar o perfil dos profissionais, envolvendo as seguintes variáveis: idade, gênero, estado civil, escolaridade, tempo de trabalho no serviço, função, jornada de trabalho e itens acerca dos riscos ocupacionais.

Os dados foram organizados em planilhas do programa Microsoft Office Excel 2010. Para a avaliação da correlação das frequências com as variáveis estabelecidas, os mesmos foram submetidos ao Teste Qui-Quadrado, com Intervalo de Confiança de 95\%, sendo estabelecida a significância em $p<0,05$, os mesmos foram transferidos para o programa estatístico R I386 3.2.2.

A pesquisa foi encaminhada para o Comitê de Ética e Pesquisa (CEP) da Faculdade Integral Diferencial - FACID/ DEVRY para apreciação, sendo aprovada em dezembro de 2015, com o número de CAAE: 46581815700005211. Foi utilizado o Termo de Consentimento Livre e Esclarecido 
(TCLE), no qual os sujeitos pesquisados foram esclarecidos sobre o estudo e principalmente sobre seus objetivos.

\section{RESULTADOS}

Os resultados apontaram o gênero feminino com uma frequência de 52,2\% (36), e o masculino com 47,8\% (33) entrevistados. A faixa etária predominante foi de 40 a 49 anos, 44,9\% (31), seguida de 30 a 39 anos, 26,1\% (18) e de 50 a 59 anos 13,0\% (9). As faixas etárias de 60 a 69 anos e de 20 a 29 anos apresentaram o mesmo número de profissionais e em menor quantidade, correspondente a 7,2\% (5).

$65,2 \%$ (45) dos profissionais são casados, 20,3\% (9) solteiros, $13,0 \%$ (9) separados e divorciados e 1,4\% (1) viúvos. Quanto à escolaridade, 36,2\% (25) possuem especialização, $33,3 \%$ (23) fizeram ensino médio, 18,8\% (13) cursaram o ensino superior, 10,1\% (7) possuem mestrado e 1,4\% (1) possui doutorado. Quanto à categoria profissional, observou-se que os técnicos de enfermagem predominam 47,8\% (33) em relação ao número de médicos $29 \%$ (20) e de enfermeiros $23,2 \%$ (16).

No que se refere ao tempo de atuação, evidenciou-se que 43,5\% (30) trabalham entre 10 a 14 anos; que 49,3\% (34) não possuem turno fixo, trabalham em ambos os turnos; $65,2 \%$ (45) não fazem turno extra de trabalho.

Em relação ao uso de equipamentos de proteção individual (EPI), todos afirmaram 100\% (69) que usam o macacão, tendo em vista que é uma exigência da instituição. Entretanto, quando se investigou o uso de outros EPIs, somente $85,5 \%$ (59) usam luvas, 84,1\% (58) máscara, 69,6\% (48) botas, 40,6\% (28) óculos, 23,2\% (16) gorros e somente $10,1 \%$ (7) utilizam todos os tipos de EPI.

Ao questionar o sentimento desses profissionais no momento do atendimento da ocorrência, 89,9\% (62) declararam-se ficar tranquilos e calmos, 5,8\% (4) declararamse tensos e apreensivos, 2,9\% estressados. As pessoas que responderam a opção intranquilo/inseguro, desconfiado e as que marcaram todas as opções apresentaram porcentagem de $1,4 \%$, respectivamente (1).

Todos os profissionais de saúde $100 \%$ (69) declararam ter consciência da existência de riscos ocupacionais ao realizar suas atividades. A tabela $\mathrm{Ol}$ apresenta os riscos físicos, de acidente de trânsito e ergonômicos. Dentre os riscos físicos, o ruído se destacou com 87,0\% (60); em relação aos riscos de acidente de trânsito a colisão prevaleceu com 79,7\% (55) e para o risco ergonômico, o levantamento de peso, no caso de maca, foi 79,7\% (55).

Tabela 01 - Riscos físicos, de acidentes e ergonômicos a que se submeteram os profissionais de saúde do SAMUTeresina-PI, no ano de 2016

\begin{tabular}{|c|c|c|c|}
\hline VARIÁVEIS & $\mathbf{N}$ & $\%$ & $\mathbf{P}$ \\
\hline \multicolumn{4}{|l|}{ Riscos fisicos } \\
\hline Ruido & 60 & $87,0 \%$ & \multirow{8}{*}{$<0,001^{* * *}$} \\
\hline Temperatura elevada & 56 & $81,2 \%$ & \\
\hline Vibrações & 29 & $42,0 \%$ & \\
\hline Frio & 22 & $31,9 \%$ & \\
\hline Radiações & 15 & $21,7 \%$ & \\
\hline Umidade & 4 & $5,8 \%$ & \\
\hline Todos & 1 & $1,4 \%$ & \\
\hline Não declarado & 3 & $4,3 \%$ & \\
\hline \multicolumn{4}{|l|}{ Risco de acidentes } \\
\hline Colisão & 55 & $79,7 \%$ & \multirow{9}{*}{$<0,001^{\star * *}$} \\
\hline $\begin{array}{l}\text { Eletricidade, incêndio } \\
\text { ou explosão }\end{array}$ & 24 & $34,8 \%$ & \\
\hline $\begin{array}{l}\text { Iluminação } \\
\text { inadequada }\end{array}$ & 23 & $33,3 \%$ & \\
\hline $\begin{array}{l}\text { Arranjo fisico } \\
\text { inadequado }\end{array}$ & 21 & $30,4 \%$ & \\
\hline $\begin{array}{l}\text { Acidentes com } \\
\text { animais }\end{array}$ & 17 & $24,6 \%$ & \\
\hline $\begin{array}{l}\text { Equipamentos } \\
\text { inadequados }\end{array}$ & 14 & $20,3 \%$ & \\
\hline Falta de proteção & 13 & $18,8 \%$ & \\
\hline Outros & 4 & $5,8 \%$ & \\
\hline Todos os descritos & 3 & $4,3 \%$ & \\
\hline \multicolumn{4}{|l|}{ Riscos ergonômicos } \\
\hline Postura inadequada & 51 & $73,9 \%$ & \multirow{7}{*}{$<0,001^{\star * *}$} \\
\hline Todos relacionados & 3 & $4,3 \%$ & \\
\hline $\begin{array}{l}\text { Levantamento de } \\
\text { peso }\end{array}$ & 55 & $79,7 \%$ & \\
\hline Esforço físico & 43 & $62,3 \%$ & \\
\hline $\begin{array}{l}\text { Controle rígido de } \\
\text { produtividade }\end{array}$ & 25 & $36,2 \%$ & \\
\hline $\begin{array}{l}\text { Monotonia e repetiti- } \\
\text { vidade }\end{array}$ & 7 & $10,1 \%$ & \\
\hline Não declarado & 1 & $1,4 \%$ & \\
\hline
\end{tabular}

A tabela 02 apresenta os riscos biológicos, químicos e psicossociais a que se submetem esses profissionais. Dentre os riscos biológicos, a contaminação por sangue predominou com 89,9\% (62); entre os riscos químicos, a poeira prevaleceu com 63,8\% (44). Em relação aos riscos psicossociais, destacou-se estresse vivenciado no momento do atendimento com 82,6\% (57); seguido do trabalho em período noturno 62,3\% (43); a elevada tensão ambiental e prejuízo na relação com colegas de trabalho, correspondem respectivamente ao mesmo percentual 40,6\% (28); jornada de trabalho prolongada 27,5\% (19), imposição de rotina intensa $15,9 \%$ (11), todos discriminados $1,4 \%$ (1) e não declarado $1,4 \%(1)$. 
Tabela 02 - Riscos biológicos, químicos e psicossociais a que se submetem os profissionais de saúde do SAMU-Teresina-PI, no ano de 2016.

\begin{tabular}{|c|c|c|c|}
\hline VARIÁVEIS & $\mathbf{N}$ & $\%$ & $\mathbf{P}$ \\
\hline \multicolumn{4}{|l|}{ Riscos biológicos } \\
\hline Sangue & 62 & $89,9 \%$ & \multirow{11}{*}{$<0,001^{\star \star \star}$} \\
\hline Liquor & 19 & $27,5 \%$ & \\
\hline Vômito & 54 & $78,3 \%$ & \\
\hline Saliva & 55 & $79,7 \%$ & \\
\hline Secreção nasal & 50 & $72,5 \%$ & \\
\hline Liquido pleural & 13 & $18,8 \%$ & \\
\hline Líquido amniótico & 40 & $58,0 \%$ & \\
\hline $\begin{array}{l}\text { Exposição a } \\
\text { perfurocortante }\end{array}$ & 42 & $60,9 \%$ & \\
\hline $\begin{array}{l}\text { Secreção advinda } \\
\text { de órgão genital }\end{array}$ & 9 & $13,0 \%$ & \\
\hline Não declarado & 1 & $1,4 \%$ & \\
\hline Todos discriminados & 9 & $13,0 \%$ & \\
\hline \multicolumn{4}{|l|}{ Riscos químicos } \\
\hline $\begin{array}{l}\text { Produtos químicos } \\
\text { (aerosóis, produtos } \\
\text { de limpeza, } \\
\text { medicamentos) }\end{array}$ & 28 & $40,6 \%$ & \multirow{7}{*}{$<0,001^{* \star *}$} \\
\hline Todos discriminados & 3 & $4,3 \%$ & \\
\hline Poeira & 44 & $63,8 \%$ & \\
\hline Fumo & 13 & $18,8 \%$ & \\
\hline Gases & 31 & $44,9 \%$ & \\
\hline Vapores & 12 & $17,4 \%$ & \\
\hline Não declarado & 6 & $8,7 \%$ & \\
\hline \multicolumn{4}{|l|}{ Riscos psicossociais } \\
\hline Situação de estresse & 57 & $82,6 \%$ & \multirow{10}{*}{$<0,001^{* * *}$} \\
\hline $\begin{array}{l}\text { Jornada de trabalho } \\
\text { prolongada }\end{array}$ & 19 & $27,5 \%$ & \\
\hline $\begin{array}{l}\text { Elevada tensão } \\
\text { ambiental }\end{array}$ & 28 & $40,6 \%$ & \\
\hline $\begin{array}{l}\text { Prejuízo na } \\
\text { inter-relação com } \\
\text { colegas de trabalho }\end{array}$ & 28 & $40,6 \%$ & \\
\hline $\begin{array}{l}\text { Prejuizo na } \\
\text { inter-relação } \\
\text { com a chefia }\end{array}$ & 13 & $18,8 \%$ & \\
\hline $\begin{array}{l}\text { Trabalho em } \\
\text { periodo noturno }\end{array}$ & 43 & $62,3 \%$ & \\
\hline $\begin{array}{l}\text { Imposição de } \\
\text { rotina intensa }\end{array}$ & 11 & $15,9 \%$ & \\
\hline Todos discriminados & 1 & $1,4 \%$ & \\
\hline Não declarado & 1 & $1,4 \%$ & \\
\hline Não declarado & 1 & $1,4 \%$ & \\
\hline
\end{tabular}

Sabe-se que a exposição aos riscos ocupacionais traz, como consequências, diversos problemas de saúde que afetam a qualidade de vida do servidor, comprometendo assim a qualidade da assistência prestada. Dentre os problemas de saúde, o distúrbio osteomuscular foi o mais relatado $34,78 \%$ (24), seguido do déficit da acuidade visual $26,09 \%$ (18), falta de apetite/fraqueza e distúrbio intestinal
20,29\% (14), distúrbio da acuidade auditiva, cansaço mental, estresse, ansiedade e depressão 17,39\% (12), dificuldade para dormir 10,14\% (7), distúrbio neuromotor e gástrico $8,70 \%$ (6), distúrbio circulatório 7,25\% (5), dor de cabeça frequente $5,80 \%$ (4), e o problema de pele, foi o menos relatado $4,35 \%$ (3). Cerca de $27,54 \%$ (19) relataram não possui, problema de saúde relacionado ao trabalho exercido.

\section{DISCUSSÃO}

Ao analisar o perfil dos trabalhadores desse serviço, pode-se constatar a predominância do sexo feminino $52,2 \%$ (36), na faixa etária entre 40 a 49 anos de idade $44,9 \%$ (31), casadas $65,2 \%$ (45). Em outras pesquisas, predominaram o sexo masculino, com idade média de 30 , na sua grande maioria, casados ${ }^{(2-3-4)}$.

Profissionais com especialização prevaleceram, 36,2\% (25). Como a graduação somente oferece uma formação generalista, enfermeiros emédicos são impulsionados a buscar formações específicas em especializações e residências. Esse é um fator positivo, uma vez que esses profissionais se apropriam de mais conhecimento e, consequentemente, podem minimizar a exposição aos riscos ocupacionais. No entanto, os técnicos de enfermagem foram a categoria profissional que mais se destacou 47,8\% (33), visto que no SAMU grande parte das ambulâncias são de Suporte Básico de Vida, composto pelo condutor e técnico de enfermagem.

Acerca do tempo de atuação, 43,5\% (30) atuam entre 10 a 14 anos, e 33,3\% (23) fazem turno extra. Em torno de 49,3\% (34) trabalham em ambos os turnos, uma vez que a jornada de trabalho do nível superior é de 24 horas semanais, com um plantão diurno e outro noturno.

Ao avaliar o uso de EPIs, todos declararam 100\% (69) usar o macacão, visto que é obrigatório o uso na instituição. As luvas foram o segundo mais usado, resultado semelhante a um estudo realizado com profissionais de enfermagem do intra-hospitalar, em que a maioria utiliza luvas (96, 5\%), seguido de máscaras (90,8\%), gorro (42,3\%) e óculos (17\%) ${ }^{(5)}$.

A maioria dos profissionais relatou sentir-se tranquila e calma no atendimento às ocorrências, o que se deve à experiência vivida durante longos anos de trabalho e a treinamentos que preparam os profissionais para lidar com diversas situações, contribuindo para sua segurança e autoconfiança.

Em relação aos riscos físicos a que são mais expostos, o ruído $87,0 \%$ (60) foi o mais frequente, em virtude da necessidade do uso da sirene na ambulância. Esse mesmo resultado foi encontrado em duas pesquisas realizadas sobre risco ocupacional em um hospital psiquiátrico de Teresina e no SAMU da região metropolitana de Natal - RN, no qual o ruido foi o risco físico $(47,6 \%)$ mais prevalente ${ }^{(1-2)}$.

A surdez é um dos distúrbios mais evidentes nos indivíduos expostos ao som de sirene de ambulância. Entretanto, existem outros riscos provocados pelo ruído como: estresse 
no atendimento ao paciente, dificuldade de concentração, dispersão, fatores que podem comprometer a saúde do trabalhador, levando ao absenteísmo(6).

Dentre o risco de acidente de trânsito, a colisão de automóveis foi a que mais acometeu a equipe 79,7\% (55). Diversos fatores a influenciam, dentre eles: sucateamento da frota de ambulância, em decorrência da reposição em tempo inábil, o mau uso da viatura pelos condutores, as "gambiarras" feitas pelas oficinas mecânicas, a qualificação inadequada dos condutores em direção defensiva. Vale ressaltar o desrespeito da população que dificulta o tráfego das ambulâncias, eventualmente ocasionando acidentes.

Dos riscos ergonômicos, o levantamento de peso, que inclui o rolamento de pacientes, levantamento de maca e de cilindros de oxigênio, se destacou com 79,7\% (55). Esse risco ocasiona os distúrbios osteomusculares, dentre os quais a lombalgia é o mais comum entre servidores desse serviço(7).

A exposição ao sangue foi o risco biológico mais relatado $89,9 \%$ (62). Pesquisa realizada com profissionais do SAMU de Minas Gerais constatou o sangue como maior risco biológico, representando 90,3\%. Esses profissionais são expostos ao sangue, principalmente, durante a imobilização do paciente (41,0\%), higienização do material (18,2\%) e intubação orotraqueal $(13,6 \%)^{(4)}$

A Organização Mundial de Saúde (OMS) estimou que 35 milhões trabalhadores de saúde, dos quais entre dois e três milhões correriam o risco de exposições percutâneas de patógenos veiculados pelo sangue a cada ano, incluindo os vírus da imunodeficiência humana (HIV), hepatite $B(H B V)$ e hepatite $\mathrm{C}(\mathrm{HCV})^{(8)}$.

Quanto aos riscos químicos, a poeira destacou-se, $63,8 \%$ (44). O resultado pode estar relacionado às condições de higiene e infraestrutura do local onde os profissionais prestam atendimento, ao não uso da máscara, pois de acordo com as variáveis, foi classificado como terceiro EPI mais utilizado, fatores que podem gerar infecções respiratórias como consequência.
O estresse vivenciado no atendimento foi o risco psicossocial que mais se submeteram 82,6\% (57). Entretanto, os casos de assédio psicológico, intimidação, assédio moral, assédio sexual e outras formas de violência estão cada vez mais presentes nesse ambiente de trabalho. Os profissionais podem adotar comportamentos nocivos à saúde para lidar com o estresse, tais como o abuso de álcool e drogas, além de serem fortes candidatos a desenvolverem doenças musculoesqueléticas, cardíacas e do sistema digestivo( ${ }^{(9)}$.

Diante do exposto, salienta-se a necessidade do desenvolvimento de atividades que melhorem a qualidade de vida e as condições de saúde e segurança do trabalhador para a garantia da assistência humanizada e integral ao cliente.

\section{CONCLUSÃO}

Os resultados apontaram uma predominância do sexo feminino, na faixa etária entre 40 a 49 anos, casadas, com atuação entre 10 a 14 anos, especialistas. O EPI mais utilizado foi o macacão, seguido das luvas e máscaras.

Os riscos a que mais se submeteram foram: ruidos da sirene de ambulância, colisão de carro, levantamento de maca, exposição a sangue, poeira ambiental e estresse no atendimento.

Sugere-se a necessidade de cursos que enfatizem a importância do uso de EPI'S e a implementação do uso do protetor auditivo, para proteger contra os ruídos inerentes ao serviço, pois é um EPI que não faz parte da rotina dos profissionais de saúde. É necessário que o gestor ofereça aos condutores curso de direção defensiva para evitar o risco de colisões. Seria interessante, também, que o serviço complementasse ao suporte psicológico as técnicas de relaxamento, pelo menos uma vez na semana, aos funcionários que compõem o serviço, com o intuído de amenizar o estresse, fator potencializador de doenças osteomusculares. Acreditase que essas iniciativas irão contribuir para minimizar a exposição dos servidores desse serviço a tais riscos.

\section{REFERÊNCIAS}

1. Fernandes MA. Marziale MHP. Riscos ocupacionais e adoecimento de trabalhadores em saúde mental.Acta paul. enferm. São Paulo. 2014; 27(6): 539-47.

2. Costa IKF, Liberato SMD, Costa IKF, Melo MDM, Simpson CA, Farias GM. Riscos ocupacionais em um serviço de atendimento móvel de urgência. Rev. de pesquisa cuidado é fundamental online. 2014; 6(3): 938 -47.

3. Gomes BB, Santos WL. Acidentes laborais entre equipe de atendimento pré - hospitalar móvel (Bombeiros/ SAMU) com destaque ao risco biológico.Rev. SenaAires.Valparaiso de Góias, GO. 2012; 1(1): 40-9.

4. Oliveira AC, Paiva MHRS.Análise dos acidentes ocupacionais com material biológico entre profissionais em serviços de atendimento pré-hospitalar.Rev. Latino-Am. Enfermagem [online]. 2013; 21(1): 309-15.

5. Santos AS, Araujo TMED, Viana MRP, Santos MS, Araujo RRMD, Campelo, TPT. Acidentes perfurocortantes em profissionais de enfermagem de serviços de urgência e emergência em uma capital brasileira. RevPesquiCuid Fundam. 2011; 3(5): 229-41.

6. Silva JLL, Costa JLS, Souza FF, Souza RL. O ruido causando danos e estresse possibilidade de atuação para a enfermagem do trabalho. av.enferm.2014; 32(1): $124-38$.

7. Schmidt DRC, Dantas RAS. Qualidade de vida no trabalho e distúrbios osteomusculares relacionados ao trabalho entre profissionais de enfermagem. Acta paul. enferm.2012:25(5): 701-7.

8. Donatelli S, Vilela RAG. Almeida IML, Reis MG.Acidente com material biológico: uma abordagem a partir da análise das atividades de trabalho. Saude soc. 2015; 24(4): 1257-72.

9. Organização Internacional do Trabalho. A prevenção das doenças profissionais 2013. 20 p. Disponivel em: <http://www.ilo.org/public/portugue/region/eurpro/ lisbon/pdf/safeday2013_relatorio.pdf>. Acesso em 4 de março de 2016. 